\title{
ALMOST COMMUTING UNITARY MATRICES
}

\author{
RUY EXEL AND TERRY LORING
}

(Communicated by John B. Conway)

\begin{abstract}
A pair of square matrices is said to be almost commuting if their commutator is small in norm. We give an elementary proof of a theorem of Voiculescu showing that not all almost commuting pairs can be perturbed to a commuting pair.
\end{abstract}

\section{INTRODUCTION}

A famous open problem popularized by Paul Halmos [3] asks whether a pair of self-adjoint matrices $A$ and $B$ which almost commute (in the sense that the operator norm $\|A B-B A\|$ is small) can always be slightly perturbed in order to yield a pair of commuting self-adjoint matrices.

While Halmos's problem remains open, several related questions have been considered and some solved. For example, if we talk about three instead of two almost commuting self-adjoints we arrive at a question which has been proved by Voiculescu [5] to be false (Davidson produces a more explicit counterexample in [2]).

The "perturbation" of Halmos's conjecture we want to consider is obtained if one replaces "self-adjoint" by "unitary" in the statement of the original problem. This turns out to be a very interesting question which attracted a fair amount of attention from several people. Again the answer is known to be false but its existing proofs are of a very interesting nature. The first counterexample, a simple one to state, was obtained by Voiculescu [6]: take $A$ as the cyclic permutation matrix and $B$ the diagonal matrix whose diagonal entries are the $n^{\text {th }}$ complex roots of the unit displayed in cyclic order.

Of course, after the above choice of matrices is granted, one is left with the task of understanding why are there no "nearby" commuting approximants.

Voiculescu remarks in his paper that his proof seems to depend on the second cohomology of the two-torus and in fact it does. In [4] the second named author

Received by the editors December 5, 1988.

1980 Mathematics Subject Classification (1985 Revision). Primary 15A15, 15A27, 47A55, 55M25; Secondary 47B47.

The first author was partially supported by $\mathrm{CNP}_{\mathrm{q}}$, Brazil.

The second author was partially supported by NSERC, Canada. 
shows an argument where the rule of the second cohomology of the two-torus comes up concretely in the form of $K$-Theory.

Choi [1] gives a proof, again in the spirit of $K$-Theory, that there exist almost commuting matrices with no commuting approximants of any form, solving a different problem from [3].

The purpose of this paper is to prove, at a completely elementary level, that there are no commuting approximants at all to Voiculescu's pair, let alone unitaries (a $K$-theoretic proof of this is given in the addendum to [4]). The "most sophisticated" tool we use is the fact that the winding number of a closed complex curve around zero is a homotopy invariant.

\section{ThE MAIN RESUlT}

Fix once and for all an integer $n \geq 7$ and let $w_{n}=e^{2 \pi i / n}$. Voiculescu's unitaries are defined by

$$
S_{n}=\left(\begin{array}{ccccc}
0 & & & & 1 \\
1 & 0 & & & \\
& 1 & 0 & & \\
& & \ddots & & \\
& & & 1 & 0
\end{array}\right) \text { and } \Omega_{n}=\left(\begin{array}{lllll}
\omega_{n} & & & & \\
& \omega_{n}^{2} & & & \\
& & \omega_{n}^{3} & & \\
& & \ddots & \\
& & & & \omega_{n}^{n}
\end{array}\right) \text {. }
$$

A few trivial and well-known facts we shall need are in order:

(a) $\left\|\Omega_{n} S_{n}-S_{n} \Omega_{n}\right\|=\left|1-\omega_{n}\right|$;

(b) $\operatorname{det}\left(\Omega_{n}\right)=\operatorname{det}\left(S_{n}\right)=(-1)^{n+1}$;

(c) $S_{n} \Omega_{n} S_{n}^{*}=\bar{\omega}_{n} \Omega_{n}$.

By (a) we have that for $n$ large, $\Omega_{n}$ and $S_{n}$ are in fact almost commuting.

Theorem. If $X$ and $Y$ are commuting $n \times n$ complex matrices then

$$
\max \left\{\left\|X-\Omega_{n}\right\|,\left\|Y-S_{n}\right\|\right\} \geq \sqrt{2-\left|1-\omega_{n}\right|}-1 .
$$

Proof. Let $X$ and $Y$ be commuting $n \times n$ matrices and let

$$
d=\max \left\{\left\|X-\Omega_{n}\right\|,\left\|Y-S_{n}\right\|\right\} .
$$

Assume by way of contradiction that $d<\sqrt{2-\left|1-\omega_{n}\right|}-1$.

For every $t$ in $[0,1]$ let $A_{t}=\Omega_{n}+t\left(X-\Omega_{n}\right)$ and $B_{t}=S_{n}+t\left(Y-S_{n}\right)$ and define $\gamma_{t}$ to be the closed complex curve given by

$$
\gamma_{t}(r)=\operatorname{det}\left((1-r) A_{t} B_{t}+r B_{t} A_{t}\right), \quad r \in[0,1] .
$$

For $t=1$ we have that $A_{t}$ and $B_{t}$ commute so $\gamma_{1}$ is a constant curve. On the other hand for $t=0$ we have $A_{t}=\Omega_{n}$ and $B_{t}=S_{n}$ hence

$$
\begin{aligned}
\gamma_{0}(r) & =\operatorname{det}\left((1-r) \Omega_{n} S_{n}+r S_{n} \Omega_{n}\right)=\operatorname{det}\left((1-r) \Omega_{n}+r S_{n} \Omega_{n} S_{n}^{*}\right) \operatorname{det}\left(S_{n}\right) \\
& =(-1)^{n+1} \operatorname{det}\left((1-r) \Omega_{n}+r \bar{\omega}_{n} \Omega_{n}\right)=(-1)^{n+1}\left(1-r+r \bar{\omega}_{n}\right)^{n} \operatorname{det}\left(\Omega_{n}\right) \\
& =\left(1-r+r \bar{\omega}_{n}\right)^{n}
\end{aligned}
$$


Note that as $r$ goes from 0 to $1,\left(1-r+r \bar{\omega}_{n}\right)$ moves along the segment joining 1 to $\bar{\omega}_{n}$ in the complex plane. It follows that $\gamma_{0}(r)$ is never zero and that it winds around zero clockwise once.

Now, since the winding number is a homotopy invariant of closed curves in the complex plane with the origin removed we shall arrive at a contradiction as soon as we prove that $\gamma_{t}(r)$ is never zero. Equivalently it suffices to show that $(1-r) A_{t} B_{t}+r B_{t} A_{t}$ is invertible for all $t$ and $r$ which we do next by proving that the latter matrix is at a distance less than one from the unitary matrix $\Omega_{n} S_{n}$.

We have

$$
\begin{aligned}
\|(1- & r) A_{t} B_{t}+r B_{t} A_{t}-\Omega_{n} S_{n} \| \\
\leq & (1-r)\left\|A_{t} B_{t}-\Omega_{n} S_{n}\right\|+r\left\|B_{t} A_{t}-\Omega_{n} S_{n}\right\| \\
\leq & (1-r)\left(\left\|A_{t} B_{t}-A_{t} S_{n}\right\|+\left\|A_{t} S_{n}-\Omega_{n} S_{n}\right\|\right) \\
& +r\left(\left\|B_{t} A_{t}-S_{n} A_{t}\right\|+\left\|S_{n} A_{t}-S_{n} \Omega_{n}\right\|+\left\|S_{n} \Omega_{n}-\Omega_{n} S_{n}\right\|\right) \\
\leq & (1-r)\left(\left\|A_{t}\right\|\left\|B_{t}-S_{n}\right\|+\left\|A_{t}-\Omega_{n}\right\|\left\|S_{n}\right\|\right) \\
& +r\left(\left\|B_{t}-S_{n}\right\|\left\|A_{t}\right\|+\left\|S_{n}\right\|\left\|A_{t}-\Omega_{n}\right\|+\left|1-\omega_{n}\right|\right) \\
\leq & (1-r)((1+d) d+d)+r\left(d(1+d)+d+\left|1-\omega_{n}\right|\right) \\
= & (1+d) d+d+r\left|1-\omega_{n}\right| \leq d^{2}+2 d+\left|1-\omega_{n}\right| .
\end{aligned}
$$

Now, since $d<\sqrt{2-\left|1-\omega_{n}\right|}-1$ we have

$$
d^{2}+2 d+\left|1-\omega_{n}\right|<1 \text {. }
$$

We do not claim that our estimate is optimal. For example, estimating $\left\|(1-r) A_{t} B_{t}+r B_{t} A_{t}-\Omega_{n} S_{n}\right\|$ for $r \leq 1 / 2$ and replacing $\Omega_{n} S_{n}$ by $S_{n} \Omega_{n}$ in a similar estimate for $r \geq 1 / 2$ we can prove that $d \geq \sqrt{2-\left|1-\omega_{n}\right| / 2}-1$.

\section{REFERENCES}

1. M. D. Choi, Almost commuting matrices need not be nearly commuting, Proc. AMS 102 (1988), 529-533.

2. K. R. Davidson, Almost commuting Hermitian matrices, Math. Scand. 56 (1985), 222-240.

3. P. R. Halmos, Some unsolved problems of unknown depth about operators on Hilbert space, Proc. Roy. Soc. Edinburgh Sect. A 76 (1976), 67-76.

4. T. A. Loring, $K$-Theory and asymptotically commuting matrices, Canad. J. Math. 40 (1988), 197-216.

5. D. Voiculescu, Remarks on the singular extension in the $C^{*}$-algebra of the Heisenberg group, J. Operator Theory 5 (1981), 147-170.

6. __ Asymptotically commuting finite rank unitary operators without commuting approximants, Acta Sci. Math. (Szeged) 45 (1983), 429-431.

Departamento de Matemática, Universidade de São Paulo, C. P. 20570, 01498 São Paulo SP, Brasil.

Department of Mathematics, Statistics and Computing Sciences, Dalhousie University, Halifax, CANada B3H $3 J 5$. 